\title{
Polarization response of second- harmonic images for different collagen spatial distributions
}

Francisco J. Ávila

Oscar del Barco

Juan M. Bueno 


\title{
Polarization response of second-harmonic images for different collagen spatial distributions
}

\author{
Francisco J. Ávila, Oscar del Barco, and Juan M. Bueno* \\ Universidad de Murcia, Instituto Universitario de Investigación en Óptica y Nanofísica, Laboratorio de Óptica, Campus de Espinardo (Ed. 34), \\ 30100 Murcia, Spain
}

\begin{abstract}
The response to polarization of second-harmonic generation (SHG) microscopy images of samples with different collagen distributions (quasialigned, partially organized, and nonorganized) has been analyzed. A linear decay relationship between the external arrangement and polarization sensitivity was found. SHG signal from nonorganized samples presented a large structural dispersion and a weak dependence with incident polarization. Polarization dependence is also associated with the internal organization of the collagen fibers, directly related to the ratio of hyperpolarizabilities $\rho$. This parameter can experimentally be computed from the modulation of the SHG signal. The results show that both external and internal collagen structures are closely related. This provides a tool to obtain information of internal properties from the polarimetric response of the external spatial distribution of collagen, which might be useful in clinical diagnosis of pathologies related to changes in collagen structure. ๑ 2016 Society of Photo-Optical Instrumentation Engineers (SPIE) [DOI: 10.1117/1.JBO.21.6.066015]
\end{abstract}

Keywords: polarization; second-harmonic generation; collagen.

Paper 160106RR received Feb. 22, 2016; accepted for publication Jun. 1, 2016; published online Jun. 22, 2016.

\section{Introduction}

The response of biological tissues to polarization can be used to explore their physical properties and to improve the visualization of details not available with other techniques. ${ }^{1,2}$ In particular, the dependence of second-harmonic generation (SHG) microscopy images of collagen-based tissues with incident polarization has been reported to better visualize certain features $^{3-5}$ and provide valuable information related to the structure and type of collagen fibers. ${ }^{6-8}$

Although it is well known that polarization modulates the spatial distribution of the SHG signal from collagen fibers, this has only been analyzed in detail for regular patterns ${ }^{9-11}$ (i.e., when the fibers are aligned along a preferential direction and the SHG signal is coherently produced). ${ }^{12}$ However, the spatial distribution is not always uniform ${ }^{13,14}$ and can be modified with aging, ${ }^{3,15,16}$ mechanical damage, ${ }^{17}$ and pathological processes $^{18-20}$ among others. In those cases, the response of the tissue to incident polarization might be noticeably different. Moreover, the SHG signal at certain locations and the visibility of details could be affected by a particular polarization state due to the spatial orientation of the collagen fibers within the sample.

In collagen type I, the fibrils forming a fiber are thought to be aligned along the fiber axis. The dipoles responsible for SHG processes are distributed within the fibrils and the total SHG signal emitted by a fiber is the coherent sum of these induced dipole moments. The molecular hyperpolarizability of collagen type I is related to the relative orientation of the fibrils and the pitch angle of the collagen triple-helix. This information can be extracted by computing the ratio of hyperpolarizabilities $\left(\rho=\beta_{x x x} / \beta_{x y y}\right) .^{5,21-23}$ It has been shown that the modulation of the SHG signal with polarization can be characterized by this ratio $\rho$, a parameter related to the internal collagen structure, ${ }^{9,11}$

*Address all correspondence to: Juan M. Bueno, E-mail: bueno@um.es which decreases with collagen aging and loss of regular distribution (i.e., denaturation). ${ }^{9,15,21,24,25}$

An objective tool to evaluate the external collagen organization (fiber distribution) in SHG images has recently been reported. This is the so-called structure tensor, ${ }^{26,27}$ which consists of a matrix of partial derivatives along the main spatial directions providing information about the orientation of every pixel of an SHG image. This procedure allows classifying the spatial distribution of collagen fibers in quasialigned, partially organized, and nonorganized.

Along this work, external organization (microscale) refers to the collagen fiber distribution that is directly quantified by applying the structure tensor to the acquired SHG images. On the other hand, the internal organization (nanoscale) is related to the distribution of the fibrils within a fiber that is characterized by the parameter $\rho$.

Although a strong polarization dependence in samples composed of quasialigned collagen fibers has been recently reported, ${ }^{11}$ an indepth analysis of the sensitivity to polarization of collagen structures with different organization is lacking in the literature. In that sense, we explore the relationship between polarization and SHG signal for samples presenting different collagen arrangements. A method to obtain the information about the internal organization from the polarization dependence of any external structure is also reported here.

\section{Methods}

\subsection{Experimental System}

A multiphoton microscope incorporating a polarization state generator (PSG) in the illumination pathway was used for the purpose of this work (Fig. 1). ${ }^{11}$ In brief, a femtosecond laser $\left(\lambda_{\omega}=760 \mathrm{~nm}, 76-\mathrm{MHz}\right.$ repetition rate) served as an illumination 


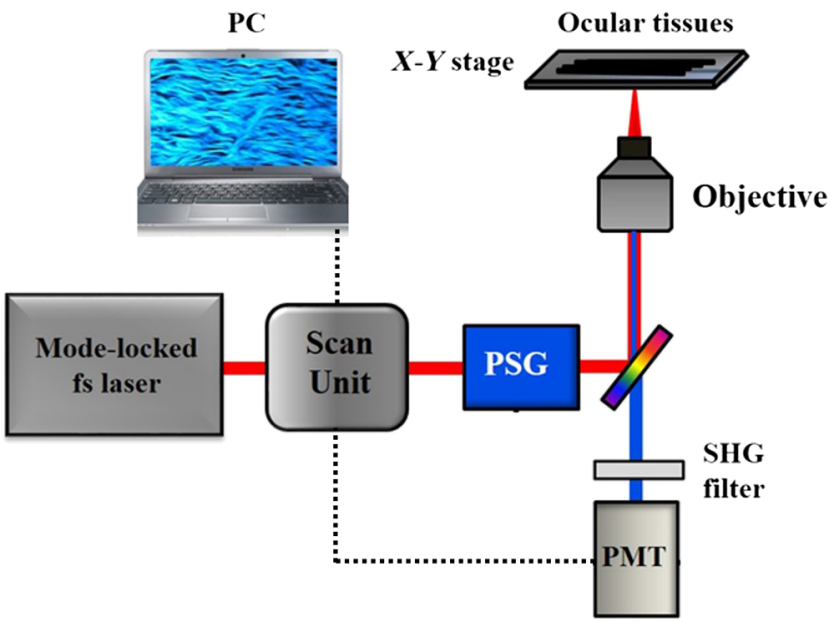

Fig. 1 Schematic diagram of the multiphoton microscope. See text for further details. PSG, polarization state generator; PMT, photomultiplier tube (see text for details).

source. The PSG was composed of a fixed horizontal linear polarizer and a rotatory half-wave plate $(\lambda / 2)$. The rotation of the $\lambda / 2$ allowed the generation of linear polarization states with different azimuth values. The polarized beam reached the sample through a nonimmersion microscope objective $[20 \times$, numerical aperture $(\mathrm{NA})=0.5]$. The nonlinear signal from the sample was collected in a backscattered configuration via the same microscope objective. A narrowband spectral filter $(380 \pm 10 \mathrm{~nm})$ placed in front of the recording unit [photomultiplier tube (PMT)] was used to isolate the SHG signal.

\subsection{Second-Harmonic Generation Microscopy Imaging Procedure}

All samples used here were nonstained ex vivo ocular tissues (nine corneas and seven sclera samples) from different species. These tissues are mainly composed of type I collagen with several arrangements. Figure 2 shows some examples of SHG images, where the different distributions of collagen fibers can easily be observed. In particular (as it will be shown in Sec. 3), these correspond to organized or quasialigned (sample \#1), partially organized (sample \#2), and nonorganized (sample \#3) collagen distribution.

For every sample, a series of polarimetric SHG images was acquired (the imaged plane was randomly chosen). Each image corresponded to a different linear polarized state produced by rotating the axis of the $\lambda / 2$ plate. Homemade MATLAB ${ }^{\mathrm{TM}}$ software was used for image processing. Figure 3 shows sets of polarimetric images for two different samples.

\subsection{Second-Harmonic Generation Response of a Set of Collagen Fibers to Incident Polarization}

A general analytical expression for the emerging SHG intensity in the backward direction $\left(\tilde{I}_{2 \omega}\right)$ from a set of $N$ collagen fibers as a function of the incident polarization has recently been reported by Ávila et al. ${ }^{11}$ In general, this equation can be expressed as

$\tilde{I}_{2 \omega}=\sum_{j=1}^{N} I_{2 \omega, j}^{\alpha_{j}} \approx A(\theta, \phi) \cdot B\left(\lambda_{\omega}, \mathrm{NA}\right) \cdot C(\chi, \psi, \rho, \mathrm{SD})$,

where $\alpha_{j}$ stands for the orientation (angle) of each collagen fiber. In Eq. (1), the first term corresponds to the SHG signal angular dependence $[(\theta, \Phi)$ are the spatial coordinates in the backward direction, i.e., $\pi / 2<\theta<3 \pi / 2$ ]. The second term depends on the excitation wavelength $\left(\lambda_{\omega}\right)$ of the incident polarized light and the NA of the microscope objective. The third term includes the azimuth $(\chi)$ and ellipticity ( $\psi$, null for linear light) of the polarized incident light, the ratio of hyperpolarizabilities of the medium $(\rho)$, and the maximum angular dispersion of the collagen fibers [i.e., the external structural dispersion (SD)].

In this model, extensively explained in Ref. 11, the set of collagen fibers was considered to present a "quasiparallel" distribution (i.e., $\mathrm{SD}=20 \mathrm{deg}$ ). For completeness, the performance and accuracy of the numerical model have been tested in collagen-based samples presenting any spatial arrangement.

\section{Results}

\subsection{Classification of the Samples According to Their Spatial Distribution}

Once the SHG images were recorded, an objective analysis of the samples to quantify the organization of the collagen structure was carried out. For this goal, the structure tensor ${ }^{26,27}$ was used. This technique provides different parameters. Among them, the histograms of orientation inform about the existence (or not) of a dominant distribution of the collagen fibers. In particular, those histograms verify that the closer the shape to a Gaussian, the higher the presence of a preferential organization. Moreover, the SD informs on the degree of organization (the larger the $\mathrm{SD}$, the lower the organization).

Figure 4 shows the histograms of preferential orientation for samples of Fig. 2. It can be observed how the Gaussian distribution vanishes from left to right. This is coherent with the
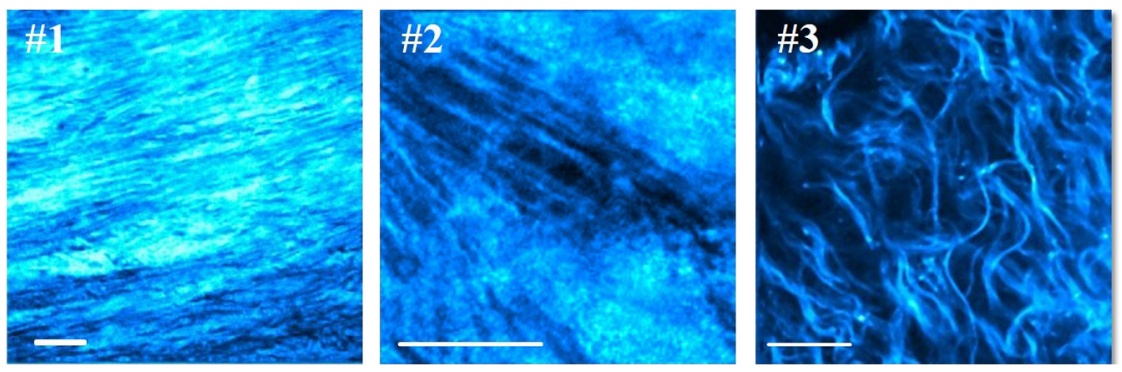

Fig. 2 Experimental SHG images corresponding to (\#1) porcine cornea, (\#2) human cornea, and (\#3) human sclera. Scale bar: $50 \mu \mathrm{m}$. 

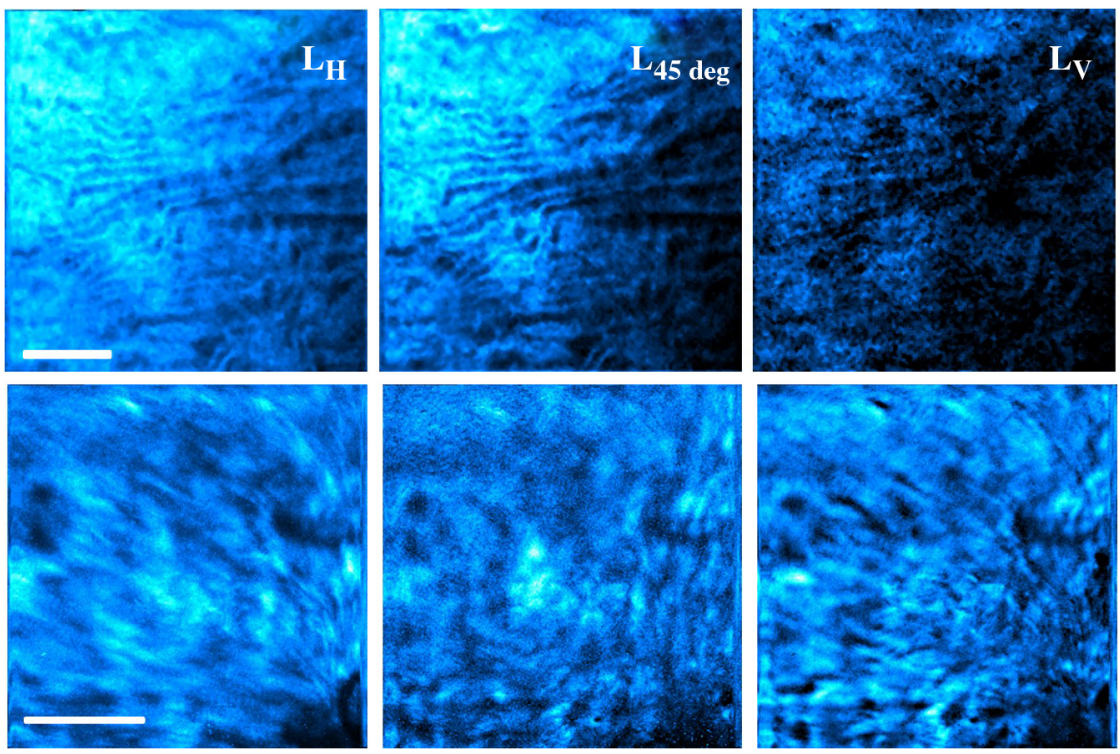

Fig. $3 \mathrm{SHG}$ images of a bovine cornea (\#4) and a bovine sclera (\#5) as a function of the incident polarization state. $L_{H}$, linear horizontal; $L_{45}$ deg, linear at $45 \mathrm{deg} ; L_{\mathrm{V}}$, linear vertical. Scale bar: $50 \mu \mathrm{m}$.
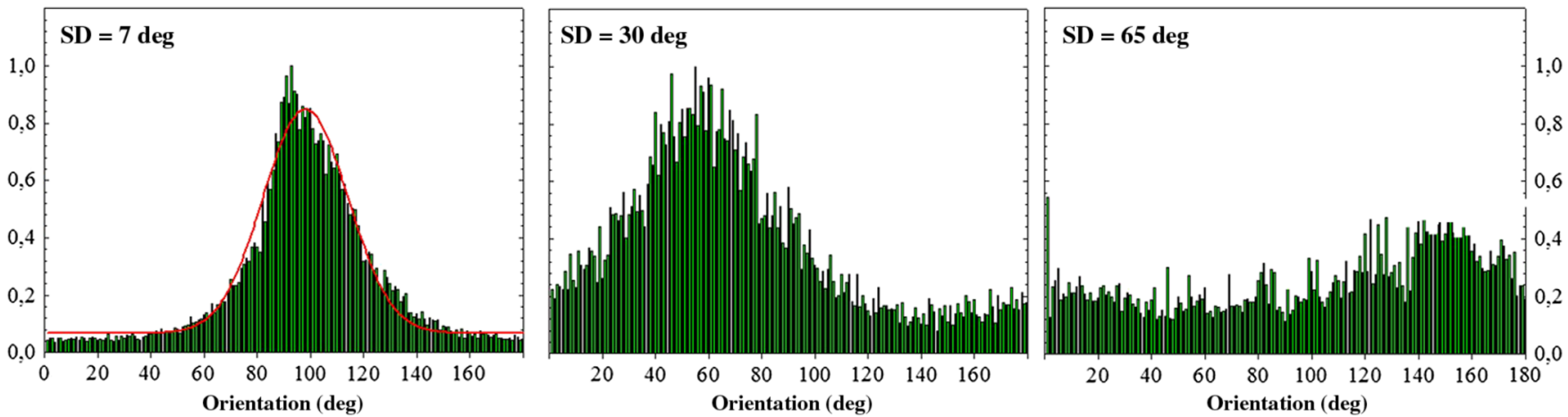

Fig. 4 Histograms of preferential orientation for samples in Fig. 2.

increase in SD values. For these samples, these values ranged between $7 \mathrm{deg}$ for the quasiorganized sample and $65 \mathrm{deg}$ for the nonorganized one. A nonparametric statistical analysis (Kolmogorov-Smirnov test) has been performed and the results revealed significant differences among them $(p<0.05)$. For the sense of completeness, Fig. 5 shows the SD for all the samples

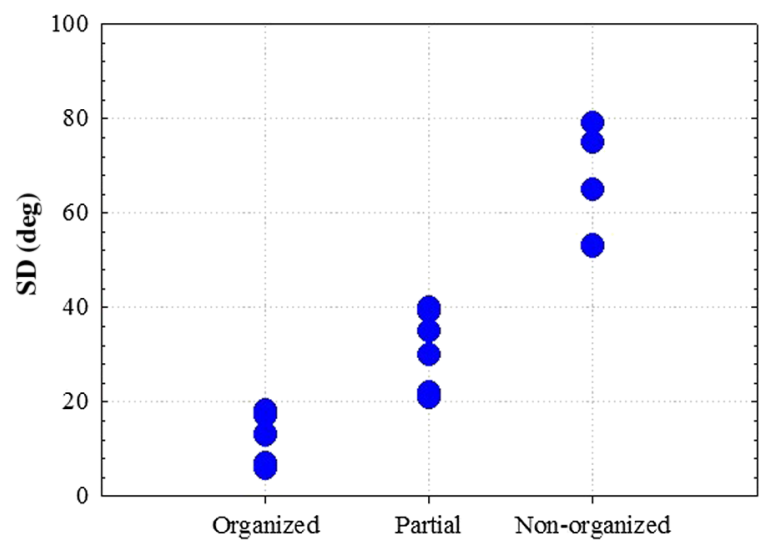

Fig. $5 \mathrm{SD}$ values and classification of the samples involved in this experiment. Quasialigned: $\mathrm{SD} \leq 20$ deg; partially organized: $20 \mathrm{deg}<\mathrm{SD} \leq 40 \mathrm{deg}$; disorganized: SD > 40 deg. involved in this study. The classification has been done according to the information reported in Ref. 27.

\subsection{Collagen External Organization and Sensitivity to Polarization}

For samples \#1, \#2, and \#3, Fig. 6(a) shows the values of total SHG intensity as a function of the azimuth of the incident linear polarized light. As a comparison, Fig. 6(b) shows the theoretical curves obtained using the model reported in Ref. 11 [i.e., via Eq. (1)]. The SD values of collagen fibers used in the simulation correspond to quasialigned (triangles), partially organized (squares), and nonorganized patterns (circles) from Fig. 4. In particular, the numerical calculation included $N=30$ collagen fibers with (SD and $\rho$ ) values of (7 deg and $-1.80 \pm 0.20)$, (30 deg and $-1.50 \pm 0.54$ ), and (65 deg and $0.49 \pm 0.19)$. The reason for the choice of these $\rho$ values is explained in Sec. 3.3.

A visual inspection reveals that the shape of the curves for samples \#1 and \#2 corresponds to specimens with negatives values of $\rho .{ }^{11}$ On the other hand, the behavior of sample \#3 is typical of a sample presenting a positive $\rho$ value. Taking into account that samples \#1 and \#2 are corneas and sample \#3 is a sclera, this agrees well with the recently reported results. ${ }^{11}$ However, those results only referred to quasiorganized samples. 


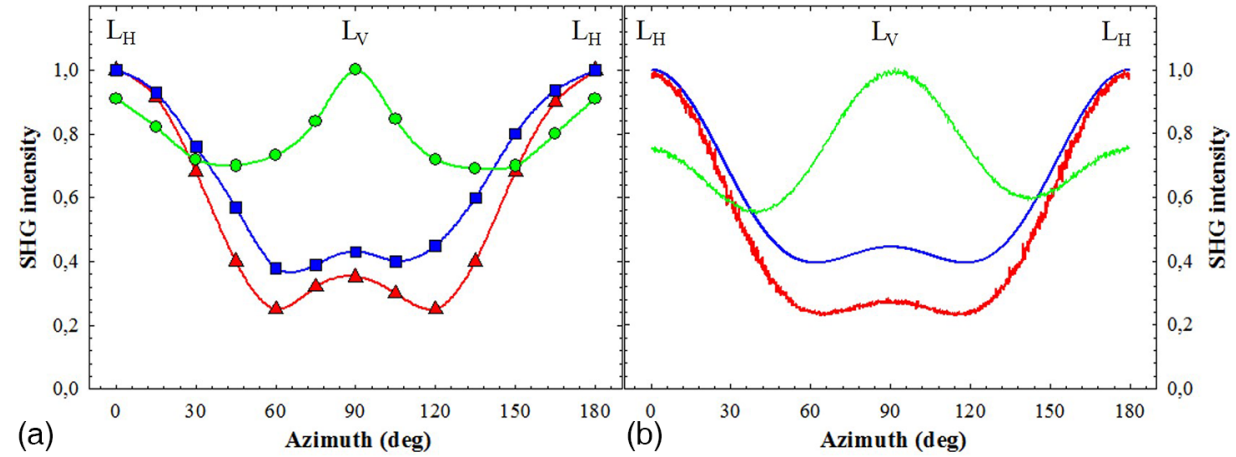

Fig. 6 (a) Experimental and (b) theoretical SHG intensity values as a function of the incident polarization (azimuth of the linear polarized states). Triangles, squares and circles correspond to samples \#1, \#2 and $\# 3$, respectively. Data have been normalized to their maxima for a better comparison. $L_{H}$, linear horizontal; $L_{\mathrm{V}}$, linear vertical.

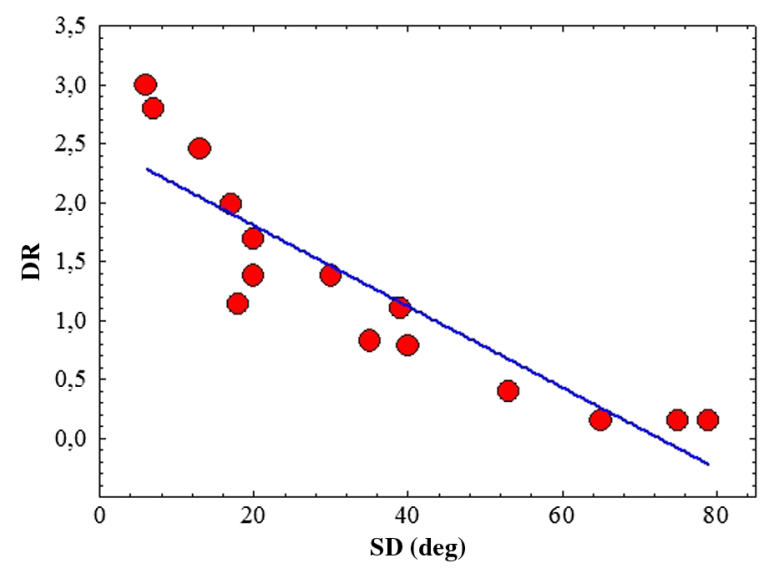

Fig. 7 Experimental DR values as function of SD for all samples here analyzed (SD values are those shown in Fig. 5). Fit equation: $\mathrm{DR}=-0.03 \cdot \mathrm{SD}+2.5$

Here, different collagen distributions have been analyzed. In fact, statistical analyses ( $t$-test) do not show significant differences when comparing Figs. 6(a) and 6(b). This indicates that the model is valid for any collagen organization and that the behavior of the SHG signal as a function of polarization provides information on the parameter $\rho$ independently on the distribution of the collagen fibers.

From data in Fig. 6, the dichroic ratio (DR) or polarization sensitivity (defined as the ratio of the intensities for linear horizontal and vertical polarization states, ${ }^{28} \mathrm{DR}=I_{\mathrm{H}} / I_{\mathrm{V}}$ ) can be calculated. In particular, DR values were $3.24,2.25$, and 0.24 for samples \#1, \#2, and \#3, respectively. That is, an organized sample might be associated to a higher DR. This is corroborated in Fig. 7, where DR values have been plotted as a function of SD for the 15 samples involved in this study. These data indicate that the SHG response of collagen-based tissues to polarization depends on the external organization of the collagen fibers (i.e., $\mathrm{SD})$. For our set of samples, a significant correlation $\left(R^{2}=0.82\right.$, $p<0.0001$ ) was found for a linear fitting.

For completeness and to corroborate the accuracy of the numerical model for different collagen organizations, Fig. 8 compares these experimental DR values with the corresponding theoretical ones for all the samples. Results reveal a statistical significant linear correlation $\left(R^{2}=0.99\right)$.

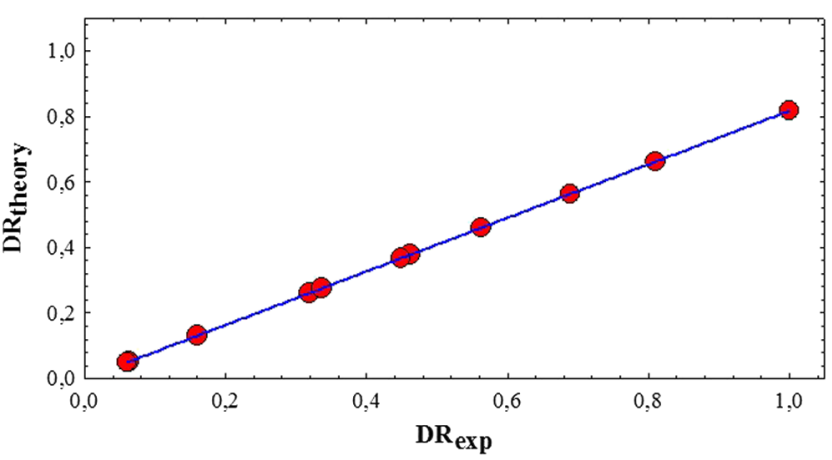

Fig. 8 Theoretical versus experimental DR values for different collagen spatial distributions.

\subsection{Relationship Between External and Internal Collagen Organization}

A strong dependence between DR and the ratio $\rho$ in samples composed of quasialigned collagen fibers has been recently reported. ${ }^{11}$ In addition, we have computed the DR value as a function of $\rho$ for different values of the SD of the collagen fibers. In particular, Fig. 9 shows the theoretical DR values as a function of $\rho$ for partially organized $(\mathrm{SD}=30 \mathrm{deg})$ and

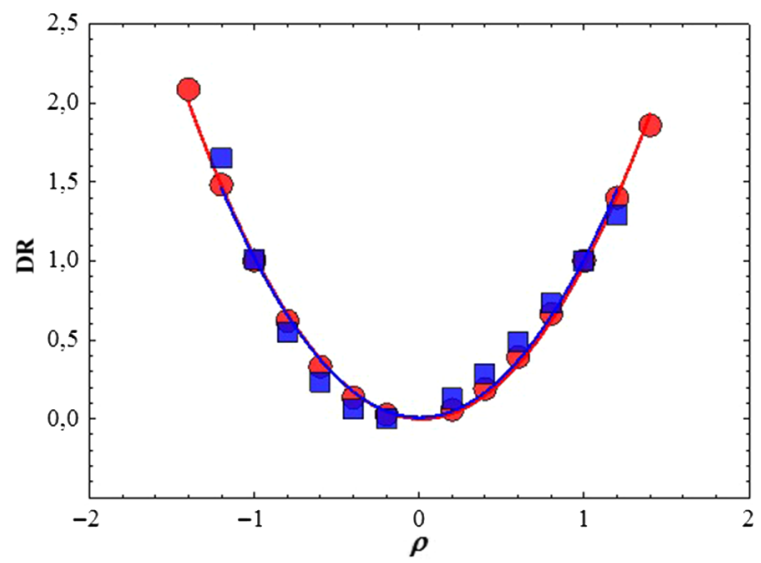

Fig. 9 Theoretical DR as a function of $\rho$ for different values of SD (partially-organized $S D=30 \mathrm{deg}$, circles; nonorganized $\mathrm{SD}=40 \mathrm{deg}$, squares). 

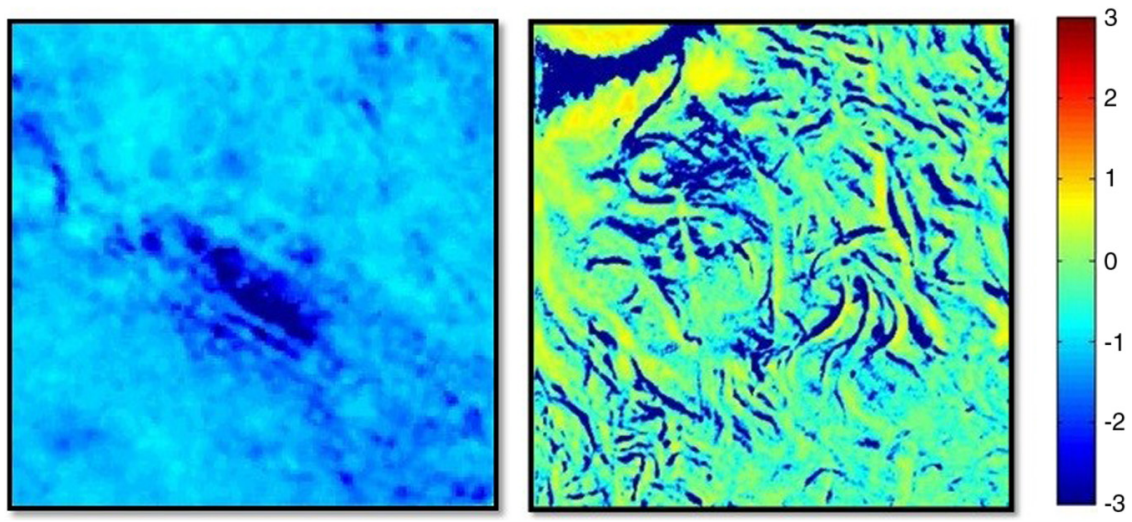

Fig. 10 Maps of parameter $\rho$ corresponding to samples \#2 (left) and \#3 (right).

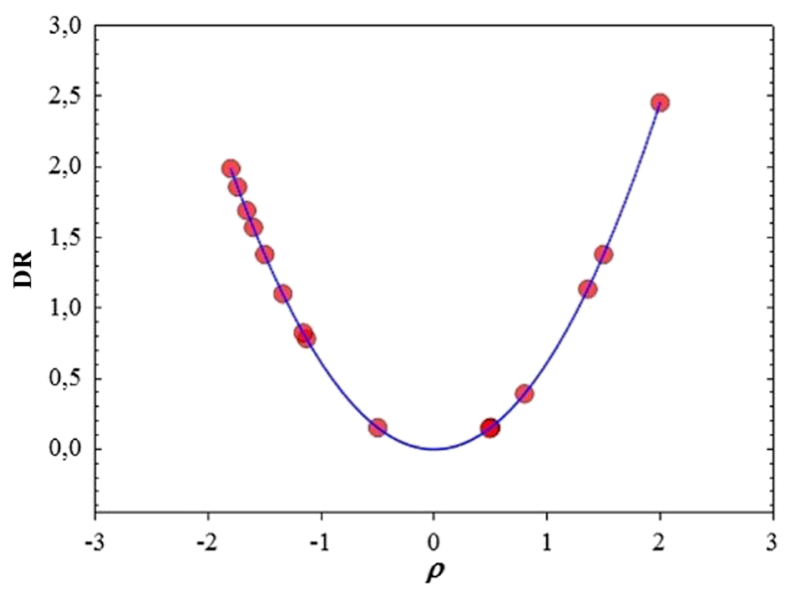

Fig. 11 Experimental DR as a function of the internal organization $(\rho)$.

nonorganized $(\mathrm{SD}=40 \mathrm{deg})$ collagen distributions. These results can be fitted to a simple parabolic curve $\mathrm{DR}=\rho^{2}\left(R^{2}=0.99\right.$ and $R^{2}=0.95$, with $\left.p<0.0001\right)$.

This parabolic fit allows us to calculate the spatially resolved values of the collagen internal organization (i.e., $\rho$ ) from the experimental values of DR, i.e., from the polarimetric SHG images corresponding to the polarization states $L_{\mathrm{H}}$ and $L_{\mathrm{v}}$. As an example, Fig. 10 shows the maps of $\rho$ for samples \#2 and \#3 computed pixel-to-pixel from the SHG images. The averaged $\rho$ values across the images were $1.50 \pm 0.54$ and $+0.49 \pm$ 0.19 [these values were used for the numerical data included in Fig. 6(b)]. The variations of the ratio $\rho$ across the image in Fig. 10 are due to the interfiber space or extracellular matrix of the scleral tissue.

The parameter $\rho$ was then computed for all samples involved in the experiment. Figure 11 shows the results. Again, these experimental data can be fitted to a parabolic curve $\left(R^{2}=\right.$ $0.99, p<0.0001)$.

At this point, it has been shown that the sensitivity to polarization of collagen-based samples (measured through DR) is related to both the external structure (i.e., SD) and the internal organization (i.e., $\rho$ ). Then, the last step would be to analyze a possible relationship between the internal organization and the external arrangement of the collagen fibers. In this sense and for all the samples analyzed along this work, Fig. 12 plots the experimental values of SD and $\rho$. As expected, both parameters are related through a parabolic relationship $\left(R^{2}=0.84, p<0.0001\right)$.

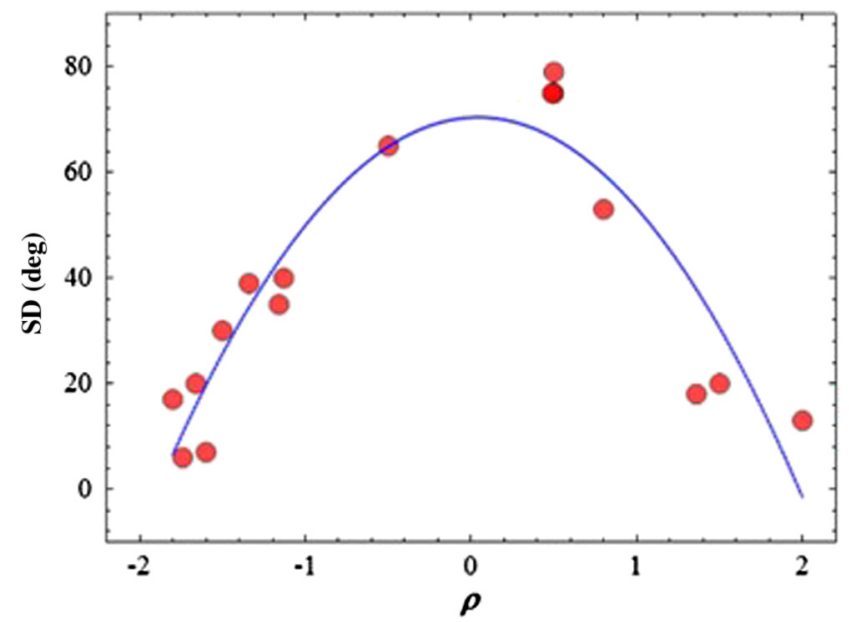

Fig. 12 Relationship between the external organization (SD) and the internal organization $(\rho)$ of the 15 collagen-based samples here analyzed. The best fit was $\mathrm{DS}=-19 \rho^{2}+1.64 \rho+70\left(R^{2}=0.84\right.$, $p<0.0001)$.

\section{Discussion and Conclusions}

There has been an increasing effort to understand the behavior of SHG signals from collagen-based tissues as a function of polarization. Moreover, interesting information on those samples has been extracted by means of polarimetric techniques combined with SHG imaging. Although it is well known that collagen fibers might present a variety of distributions, ${ }^{13,14}$ numerical models were only able to explain the behavior of sets of quasialigned fibers. ${ }^{1,9,11}$

This work dealt with the analysis of the SHG signal response to polarization for different organizations of the collagen fibers. This collagen distribution of the samples has been classified by means of the structure tensor. ${ }^{11}$ Although the specimens analyzed were healthy ocular tissues (cornea and sclera), the results can be extended to any collagen-based sample. Depending on the type of tissue, the histograms of preferential orientation provided by the structure tensor revealed different collagen distributions. Unlike the sclera that always presented a nonorganized structure, the cornea showed both quasialigned and partially organized collagen fibers.

This objective classification of collagen distribution allowed an accurate analysis of the response to incident linear polarization. Independently on the type of organization, the SHG signal presented a periodic shape [Fig. 6(a)]. This shape was similar for 
quasialigned and partially aligned samples, showing a secondary maximum with noticeable lower intensity than the primary one. However, it changed dramatically for nonorganized distributions, where both maxima SHG intensity values were similar.

The dependence between the modulation of the SHG signal (computed as the DR) and collagen external arrangement (computed via the SD) was shown in Fig. 7. A statistically significant linear correlation was found between both the parameters. This behavior indicates that there is a strong relationship between the collagen distribution and the response to incident polarization. In general, it could be claimed that the more organized the sample, the stronger the modulation. In fact, nonorganized samples hardly depend on the incident polarization.

Ávila et al. ${ }^{11}$ recently reported a numerical model to analyze the dependence between the SHG intensity and the incident polarization for samples composed of elongated collagen fibers. Since statistical differences between experimental and theoretical data were not found, the model is also valid for sets of fibers with any spatial distribution. For each sample involved in the experiment, this fact allowed a reliable approach to determine the (spatially resolved) values of $\rho$. In particular, our averaged value for a human cornea $(\rho=-1.50)$ fairly agrees with that calculated by Latour et al. $(|\rho|=1.44) .{ }^{29}$ Moreover, results show a parabolic dependence between the DR and the parameter $\rho$ (Figs. 9 and 11). This means that there also exits a strong dependence between the collagen internal organization and the polarization sensitivity, the higher the $|\rho|$, the higher the DR.

The parameter $\rho$ plays a fundamental role in characterizing the collagen structure, and the polarization response of the SHG signal depends on its sign. ${ }^{11}$ At this point, it is also interesting to notice that the shape of the curves of polarization sensitivity confirms that $\rho$ is positive for the sclera and negative for the cornea. The former corresponds to a dense irregular connective tissue, whereas the latter is a dense regular one. The information obtained from the parameter $\rho$ is very valuable to understand and characterize collagen nature, whereas the module is related to the internal organization, the sign would inform about the type of connective tissue.

However, probably the most interesting result reported is the possibility to establish an experimental relationship between external and internal collagen organizations (as shown in Fig. 12). This means that, in a collagen-based sample, the arrangements at both scales are not independent. It has been shown that low values of $|\rho|$ are related with a high SD (and conversely). That is, from an experimental point of view, it would not be possible to find a sample with both low (high) internal organization and low (high) SD of the fibers.

In conclusion, the sensitivity to polarization of SHG images of collagen-based samples has been explored. An linear relationship with the SD was found. Unlike quasialigned samples, nonorganized collagen distributions hardly depend on incident polarization. For partially organized specimens, the modulation depends on their SD. From this dependence between SHG and polarization, the value of $\rho$ can also be derived, which provides information on the internal structure directly obtained from the SHG external polarimetric response. Moreover, the parameter $\rho$ and the external SD were found to be related through a parabolic function. This can be used as a powerful tool in clinical diagnosis, since many pathologies of the connective tissue, such as cancer, ${ }^{30}$ might present early signs at internal scale (collagen denaturation) before external changes in the organization in the collagen fibers occur.

\section{Acknowledgments}

This work was supported by the Secretaría de Estado de Investigación, Desarrollo e Innovación, Spain (Grant No. FIS2013-41237-R).

\section{References}

1. R. M. Williams, W. R. Zipfel, and W. W. Webb, "Interpreting secondharmonic generation images of collagen I fibrils," Biophys. J. 88(2), 1377-1386 (2005).

2. J. M. Bueno et al., "Improved scanning laser fundus imaging using polarimetry," J. Opt. Soc. Am. A. 24(5), 1337-1348 (2007).

3. P. Stoller, K. M. Reiser, and P. M. Celliers, "Polarization-modulated second harmonic generation in collagen," Biophys. J. 82(6), 33303342 (2002).

4. C. K. Chou et al., "Polarization ellipticity compensation in polarization second-harmonic generation microscopy without specimen rotation," J. Biomed. Opt. 13(1), 014005 (2008).

5. I. Gusachenko, G. Latour, and M. C. Schanne-Klein, "Polarization resolved second harmonic microscopy in anisotropic thick tissues," Opt. Express 18(18), 19339-19352 (2010).

6. P. Stoller et al., "Quantitative second-harmonic generation microscopy in collagen," Appl. Opt. 42(25), 5209-5219 (2003).

7. A. T. Yeh, N. Nassif, and B. J. Tromberg, "Selective corneal imaging using combined second-harmonic generation and two-photon excited fluorescence," Opt. Lett. 27(23), 2082-2084 (2002).

8. K. Tilbury et al., "Differentiation of Col I and Col III isoforms in stromal models of ovarian cancer by analysis of second harmonic generation polarization and emission directionality," Biophys. J. 106(2), 354-365 (2014).

9. Y. Chang et al., "Theoretical simulation study of linearly polarized light on microscopic second-harmonic generation in collagen type I," J. Biomed. Opt. 14(4), 044016 (2009).

10. O. del Barco and J. M. Bueno, "Second harmonic generation signal in collagen fibers: role of polarization, numerical aperture, and wavelength," J. Biomed. Opt. 17(4), 045005 (2012).

11. F. J. Ávila, O. del Barco, and J. M. Bueno, "Polarization dependence of aligned collagen tissues imaged with second harmonic generation microscopy," J. Biomed. Opt. 20(8), 086001 (2015).

12. P. N. Butcher and D. Cotter, The Elements of Nonlinear Optics, Vol. 9, Cambridge University Press, Cambridge, United Kingdom (1990).

13. J. M. Bueno, E. J. Gualda, and P. Artal, "Analysis of corneal stroma organization with wavefront optimized nonlinear microscopy," Cornea 30(6), 692-701 (2011).

14. J. M. Bueno et al., "Analysis of spatial lamellar distribution from adaptive-optics second harmonic generation corneal images," Biomed. Opt. Express 4(7), 1006-1013 (2013).

15. I. Freund, M. Deutsch, and A. Sprecher, "Connective tissue polarity: optical second-harmonic microscopy, crossed-beam summation, and smallangle scattering in rat-tail tendon," Biophys. J. 50(4), 693-712 (1986).

16. S. Wu et al., "Quantitative analysis on collagen morphology in aging skin based on multiphoton microscopy," J. Biomed. Opt. 16(4), 040502 (2011).

17. P. Matteini et al., "Photothermally-induced disordered patterns of corneal collagen revealed by SHG imaging," Opt. Express 17(6), 4868-4878 (2009).

18. M. M. Johns et al., "Radiation fibrosis of the vocal fold: from man to mouse," Laryngoscope 122(5), S107-S125 (2012).

19. M. Hadian et al., "Collagen organization in canine myxomatous mitral valve disease: an x-ray diffraction study," Biophys. J. 93(7), 2472-2476 (2007).

20. P. J. Campagnola, "Second harmonic generation imaging microscopy: applications to diseases diagnostics," Anal. Chem. 83(9), 3224-3231 (2011).

21. S. Roth and I. Freund, "Second-harmonic generation in collagen," J. Chem. Phys. 70(4), 1637-1643 (1979).

22. P. J. Su et al., "Discrimination of collagen in normal and pathological skin dermis through second-order susceptibility microscopy," Opt. Express 17(13), 11161-11171 (2009).

23. P. Stoller et al., "Quantitative second-harmonic generation microscopy in collagen," Appl. Opt. 42(25), 5209-5219 (2003). 
24. C. Odin et al., "Orientation fields of nonlinear biological fibrils by second harmonic generation microscopy," J. Microsc. 229(1), 32-38 (2008).

25. P. Stoller et al., "Polarization-dependent optical second-harmonic imaging of a rat-tail tendon," J. Biomed. Opt. 7(2), 205-214 (2002).

26. B. Jahne, Spatio-Temporal Image Processing: Theory and Scientific Applications, Springer, Berlin (1993).

27. F. J. Ávila and J. M. Bueno, "Analysis and quantification of collagen organization with the structure tensor in second harmonic microscopy images of ocular tissues," Appl. Opt. 54(33), 9848-9854 (2015).
28. D. Marsh, "Dichroic ratios in polarized Fourier transform infrared for non-axial symmetry of $\beta$-sheet structures," Biophys. J. 72(6), 27102718 (1997).

29. G. Latour et al., "In vivo structural imaging of the cornea by polarization-resolved second harmonic microscopy," Biomed. Opt. Express 3(1), 1-15 (2012).

30. F. Barclay, F. Arcuri, and A. Paparatto, "Manguito rotador: histología de la normalidad a la patología," Artroscopia 18(1), 30-36 (2011).

Biographies for the authors are not available. 